\title{
Optimal Multicarrier Phase-Coded Waveform Design for Detection of Extended Targets ${ }^{\dagger}$
}

\author{
Satyabrata Sen and Charles W. Glover \\ Center for Engineering Science Advanced Research \\ Computer Science and Mathematics Division \\ Oak Ridge National Laboratory \\ 1 Bethel Valley Road, Oak Ridge, TN 37831-6015, USA \\ Email: \{sens, glovercw\} @ornl.gov_ Phone: 865-241-9117
}

\begin{abstract}
We design a parametric multicarrier phase-coded (MCPC) waveform that achieves the optimal performance in detecting an extended target in the presence of signal-dependent interference. Traditional waveform design techniques provide only the optimal energy spectral density of the transmit waveform and suffer a performance loss in the synthesis process of the timedomain signal. Therefore, we opt for directly designing an MCPC waveform in terms of its time-frequency codes to obtain the optimal detection performance. First, we describe the modeling assumptions considering an extended target buried within the signal-dependent clutter with known power spectral density, and deduce the performance characteristics of the optimal detector. Then, considering an MCPC signal transmission, we express the detection characteristics in terms of phase-codes of the MCPC waveform and propose to optimally design the MCPC signal by maximizing the detection probability. Our numerical results demonstrate that the designed MCPC signal attains the optimal detection performance and requires a lesser computational time than the other parametric waveform design approach.
\end{abstract}

\section{INTRODUCTION}

The problem of waveform design is becoming increasingly relevant and challenging to the modern state-of-the-art radar systems. For many years, the conventional radars have transmitted a fixed waveform on every pulse, and the research efforts have been primarily devoted to optimally process the corresponding received signals [1]. However, with the recent technological advancements in the fields of flexible waveform generators and high-speed signal processing hardware, it is now possible to generate and transmit sophisticated radar waveforms that are optimally adapted to the sensing environments on a periodic basis (potentially on a pulse-by-pulse basis) [2]-[6]. Such adaptation can lead to a significant performance gain over the classical (non-adaptive) radar waveforms, particularly in the defense applications involving fast-changing scenarios.

Most of the salient research works on waveform design have addressed the problem of target detection and classification [7]-[11]. They can primarily be distinguished in terms of the employed optimality-criterion, e.g., signal-to-noise ratio (SNR), mutual information (MI), detection probability, as well as by the assumed modeling-conventions of the target and interference scenario, e.g., point or extended targets, deterministic or stochastic target-response, and signal-dependent

$\dagger$ This research was partially supported by the U.S. Missile Defense Agency (MDA) at the Oak Ridge National Laboratory, managed by UT-Battelle, LLC, for the U.S. Department of Energy, under contract DE-AC05-00OR22725. or signal-independent interference. The commonality among them can be noticed in the design results which are provided in terms of the optimal energy-spectral-density (ESD) of the transmit signal. However, it has been shown that a timedomain synthesis of the transmit signal to achieve the optimal ESD suffers a considerable performance loss [12]. Therefore, in recent times, [12], [13] propose to directly design a parametric time-domain signal that achieves the optimal detection performance; our work also belongs to this parametric signal design paradigm.

The contribution of this work is to design a parametric multicarrier phase-coded (MCPC) waveform that attains the optimal performance in detecting an extended target. The MCPC signal is found to have many advantages in the radar applications compared to other phase-coded signals [14]-[16, Ch. 11]. It has higher spectral efficiency than a multicarrier signal based on the linear frequency modulation (LFM). Unlike the P3 and P4 signals, MCPC has a thumbtack ambiguity function and requires fewer phase values. Additionally, being a multicarrier signal, an MCPC waveform resulted from an efficient frequency-based design can significantly reduce the autocorrelation sidelobes.

First, in Section II, we describe the system models including an extended target in the presence of signal-dependent clutter and noise. This is followed by the derivation of the optimum Neyman-Pearson detector and its performance characteristics. Then, in Section III, we provide a short review of the MCPC waveforms and formulate an optimization problem to design the phase-codes of an MCPC waveform in order to achieve the optimal detection performance. We present numerical examples in Section IV to compare the performances of the designed MCPC signal with those of the optimal-ESD generation approach [8], [11], and an existing waveform design technique based on the discrete prolate spheroidal sequences (DPSS) [12]. Our results demonstrate that the design of MCPC signal requires considerably less computational-time than that for the DPSS waveforms and provides the optimal detection performance along with a much clear time-frequency representation of the designed transmit signal.

\section{PROBLEM DESCRIPTION}

Fig. 1 depicts a simple, schematic representation of a generic radar system designed for a target detection problem. We 


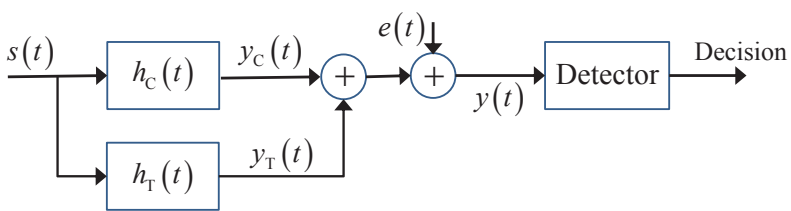

Fig. 1. A schematic block-diagram of a generic radar model.

denote the complex envelope of the transmitted signal by $s(t)$. We consider $s(t)$ as an energy-limited and finite-duration signal, which is nonzero only when $0 \leq t \leq T$ and has the total transmitted energy $E_{\mathrm{s}}=\int_{0}^{T}|s(t)|^{2} d t$. The target and clutter scattering are modeled with two linear and timeinvariant impulse responses $h_{\mathrm{T}}(t)$ and $h_{\mathrm{c}}(t)$, respectively. Therefore, for a transmitted signal $s(t)$, the target and clutter responses are respectively manifested at the radar receiver as $y_{\mathrm{T}}(t)=h_{\mathrm{T}}(t) * s(t)$ and $y_{\mathrm{c}}(t)=h_{\mathrm{c}}(t) * s(t)$, where $*$ denotes convolution in time. In addition, $e(t)$ represents the measurement error that in general includes the receiver thermal-noise and any passive-interferences (jamming).

We further assume that the clutter and noise processes are complex, wide-sense stationary (WSS), zero-mean Gaussian random processes with known power spectral densities (PSDs) $\mathcal{P}_{\mathrm{c}}(f)$ and $\mathcal{P}_{\mathrm{e}}(f)$ respectively. In the literature, the target response is considered as both the deterministic and stochastic processes; in this work, we assume the Swerling-I type target model $h_{\mathrm{T}}(t)=x_{\mathrm{T}} g_{\mathrm{T}}(t)$, where $x_{\mathrm{T}}$ is assumed to be a complex, zero-mean Gaussian random variable with variance $\sigma_{\mathrm{x}}^{2}$; and $g_{\mathrm{T}}(t)$ is a known deterministic function that depends on the target orientation angle and radar aspect angle. Based on these assumptions and also considering mutual independence among the target, clutter, and noise processes, the received signal $y(t)$ becomes a complex, zero-mean Gaussian process with PSD either $\mathcal{P}_{y \mid \mathcal{H}_{0}}(f)=\mathcal{P}_{\mathrm{c}}(f)|S(f)|^{2}+\mathcal{P}_{\mathrm{e}}(f)$ or $\mathcal{P}_{y \mid \mathcal{H}_{1}}(f)=\sigma_{\mathrm{x}}^{2}\left|G_{\mathrm{T}}(f)\right|^{2}|S(f)|^{2}+\mathcal{P}_{y \mid \mathcal{H}_{0}}(f)$, depending on the absence or presence of the target. Here, $G_{\mathrm{T}}(f), S(f)$ respectively are the Fourier transforms of $g_{\mathrm{T}}(t), s(t)$; and $\mathcal{H}_{0}$, $\mathcal{H}_{1}$ denote the null (target-free) and alternate (target-present) hypotheses.

Next, we convert the received signal into the frequency domain using the Fourier transform. Considering $B$ as the total bandwidth and $K$ as the number of discrete frequency-bins with the frequency spacing $\Delta f=B / K$, we denote $Y\left(f_{k}\right)$ to be the frequency sample of $y(t)$ at the $k$ th frequency-bin and write the detection problem as

$$
\begin{array}{ll}
\mathcal{H}_{0}: & \boldsymbol{Y}=\boldsymbol{Y}_{\mathrm{c}}+\boldsymbol{E} \\
\mathcal{H}_{1}: & \boldsymbol{Y}=x_{\mathrm{T}} \boldsymbol{Y}_{\mathrm{T}}+\boldsymbol{Y}_{\mathrm{c}}+\boldsymbol{E},
\end{array}
$$

where $\boldsymbol{Y}=\left[Y\left(f_{(-K / 2)}\right), \ldots, Y\left(f_{(K / 2-1)}\right)\right]^{T}$, and similarly $\boldsymbol{Y}_{\mathrm{T}}, \boldsymbol{Y}_{\mathrm{c}}$, and $\boldsymbol{E}$ include all the frequency samples of $\left(g_{\mathrm{T}}(t) * s(t)\right), \quad\left(h_{\mathrm{c}}(t) * s(t)\right)$, and $e(t)$ respectively. The statistical properties of the target, clutter, and noise processes mentioned earlier enable us to have

$$
\begin{array}{ll}
\mathcal{H}_{0}: & \boldsymbol{Y} \sim \mathbb{C N}\left(\mathbf{0}, \boldsymbol{R}_{0}\right), \\
\mathcal{H}_{1}: & \boldsymbol{Y} \sim \mathbb{C N}\left(\mathbf{0}, \boldsymbol{R}_{1}\right)
\end{array}
$$

where $\boldsymbol{R}_{0}$ asymptotically becomes a diagonal matrix for large time-bandwidth product $T B$ with

$$
\left[\boldsymbol{R}_{0}\right]_{(k, k)}=\mathcal{P}_{y \mid \mathcal{H}_{0}}\left(f_{k}\right),
$$

and $\boldsymbol{R}_{1}$ can be expressed as

$$
\boldsymbol{R}_{1}=\boldsymbol{R}_{0}+\sigma_{\mathrm{x}}^{2} \boldsymbol{Y}_{\mathrm{T}} \boldsymbol{Y}_{\mathrm{T}}^{H} .
$$

Under this circumstances, following the similar formulations as in [8], [12], we can characterize the performance of the optimum Neyman-Pearson detector in terms of the probabilities of detection and false-alarm as

$$
P_{\mathrm{D}}=P_{\mathrm{FA}}^{\left[1 /\left(1+d^{2}\right)\right]},
$$

where

$$
\begin{aligned}
d^{2} & =\sum_{k=-K / 2}^{K / 2-1} \frac{\sigma_{\mathrm{x}}^{2}\left|G_{\mathrm{T}}\left(f_{k}\right)\right|^{2}\left|S\left(f_{k}\right)\right|^{2}}{\mathcal{P}_{\mathrm{c}}\left(f_{k}\right)\left|S\left(f_{k}\right)\right|^{2}+\mathcal{P}_{\mathrm{e}}\left(f_{k}\right)} \Delta f, \\
& \approx \int_{-B / 2}^{B / 2} \frac{\sigma_{\mathrm{x}}^{2}\left|G_{\mathrm{T}}(f)\right|^{2}|S(f)|^{2}}{\mathcal{P}_{\mathrm{C}}(f)|S(f)|^{2}+\mathcal{P}_{\mathrm{e}}(f)} d f .
\end{aligned}
$$

Therefore, for a given $P_{\mathrm{FA}}, P_{\mathrm{D}}$ can be made larger by increasing $d^{2}$. Thus, an optimal waveform that produces maximum $d^{2}$ value can be expressed in terms of its ESD, $\mathcal{E}_{\mathrm{s}}(f) \triangleq|S(f)|^{2}$, as

$\mathcal{E}_{\mathrm{s}, \text { opt }}\left(f_{k}\right)=\max \left(\frac{\sigma_{\mathrm{x}}\left|G_{\mathrm{T}}\left(f_{k}\right)\right| \sqrt{\mathcal{P}_{\mathrm{e}}\left(f_{k}\right) / \lambda}-\mathcal{P}_{\mathrm{e}}\left(f_{k}\right)}{\mathcal{P}_{\mathrm{c}}\left(f_{k}\right)}, 0\right)$,

where $\lambda$ is computed to satisfy a pre-defined total-energy constraint $\varepsilon$, i.e., $E_{\mathrm{s}}=\sum_{k=-K / 2}^{K / 2-1} \mathcal{E}_{\mathrm{s}, \mathrm{opt}}\left(f_{k}\right) \Delta f=\varepsilon$. This implies that the optimal-ESD, $\mathcal{E}_{\mathrm{s}, \text { opt }}$, is nonzero only at those frequency-bins in which the target power is greater than the scaled noise power $\lambda \mathcal{P}_{\mathrm{e}}(f)$ (termed as the water level).

\section{Signal Modeling ANd WaVeform Design}

In this section, we develop an algorithm to directly synthesize a transmit waveform $s(t)$ that achieves the optimal detection performance expressed in terms of $d^{2}$ of (6). Mathematically, obtaining the optimal transmit signal $s(t)$ is tantamount to finding the optimal design of an $L \times 1$ signal-vector $s=[s(0), s(1), \ldots, s(L-1)]^{T}$, where $\{s(l) \equiv s(t=$ $\left.\left.l t_{\mathrm{s}}\right), l=0,1, \ldots, L-1\right\}$, with the total-energy requirement $E_{\mathrm{S}}=t_{\mathrm{S}} \boldsymbol{s}^{H} \boldsymbol{s}=\varepsilon$. Here, $t_{\mathrm{S}}=T / L \leq 1 /(2 B)$ denotes the sampling interval, and hence we require at least $L=2 T B$ temporal samples.

Representing the frequency response of the transmit signal at the $k$ th frequency-bin as

$$
S\left(f_{k}\right)=\boldsymbol{w}_{k}^{H} \boldsymbol{s},
$$

where $\boldsymbol{w}_{k}^{H}=\sqrt{\frac{t_{\mathrm{s}}}{B}}\left[e^{-j(2 \pi / K)(k .0)}, \ldots, e^{-j(2 \pi / K)(k .(L-1))}\right]$ is an $1 \times L$ vector representing a scaled version of the $k$ th row of the discrete Fourier transform matrix, we can rewrite the optimal detection performance of (6) as

$$
d(\boldsymbol{s})^{2}=\sum_{k=-K / 2}^{K / 2-1} \frac{\boldsymbol{s}^{H}\left[\sigma_{\mathrm{x}}^{2}\left|G_{\mathrm{T}}\left(f_{k}\right)\right|^{2} \boldsymbol{w}_{k} \boldsymbol{w}_{k}^{H}\right] \boldsymbol{s}}{\boldsymbol{s}^{H}\left[\mathcal{P}_{\mathrm{c}}\left(f_{k}\right) \boldsymbol{w}_{k} \boldsymbol{w}_{k}^{H}\right] \boldsymbol{s}+\mathcal{P}_{\mathrm{e}}\left(f_{k}\right)} \Delta f .
$$


Subsequently, we can obtain the optimal transmit signal by maximizing $d^{2}$ with respect to $s$ as

$$
\boldsymbol{s}_{\mathrm{U}}=\arg \max _{\boldsymbol{s} \in \mathbb{C}^{L}} d(\boldsymbol{s})^{2} \quad \text { subject to } E_{\mathrm{s}}(\boldsymbol{s})=\varepsilon,
$$

where $E_{\mathrm{s}}(\boldsymbol{s})=\sum_{k=-K / 2}^{K / 2-1}\left[\boldsymbol{s}^{H} \boldsymbol{w}_{k} \boldsymbol{w}_{k}^{H} \boldsymbol{s} \Delta f\right]=t_{\mathrm{s}} \boldsymbol{s}^{H} \boldsymbol{s}$.

We refer to the solution of (11) as an unstructured waveform $s_{\mathrm{U}}$, because it does not have any particular form or composition. However, oftentimes the synthesized signals belong to a certain class or family of waveforms having well-defined structures. For example, considering only the linear transformations, we can construct a signal $s_{\Phi}$ from a family of waveforms $\mathfrak{S}_{\Phi}$ as

$$
\boldsymbol{s}_{\Phi} \in \mathfrak{S}_{\Phi}:=\{\boldsymbol{s} \mid \boldsymbol{s}=\boldsymbol{\Phi} \boldsymbol{z}\},
$$

where $\boldsymbol{\Phi}$ is an $L \times P$ matrix whose fixed composition defines the family $\mathfrak{S}_{\Phi}$; and $z$ is a $P \times 1$ code vector whose entries can be varied to generate different realizations of $s_{\Phi}$.

Examples of transmit signals having the form of (12) include the MCPC waveforms. An MCPC pulse consists of $N$ carriers that simultaneously transmit $N$ sequences each of which is phase modulated by $M$ bits in time. Defining one bit duration as $t_{\mathrm{b}}=T / M=(L / M) t_{\mathrm{s}}$, the complex envelope of an MCPC pulse is given by

$s(l)=\sum_{m=1}^{M}\left[\sum_{n=1}^{N} a_{m, n} e^{j 2 \pi\left(n-\frac{N+1}{2}\right) \frac{l t_{\mathrm{s}}}{t_{\mathrm{b}}}}\right] q\left(l t_{\mathrm{s}}-(m-1) t_{\mathrm{b}}\right)$,

where $a_{m, n}$ is the complex code modulating the $n$th carrier at the $m$ th bit-duration; and $q(t) \equiv 1$ for $0 \leq t \leq t_{\mathrm{b}}$ and zero elsewhere. Here, the carriers are equally spaced with the frequency difference equal to the inverse of the bit-duration, and therefore an MCPC signal occupies a bandwidth $B=$ $N / t_{\mathrm{b}}$ and has a time-bandwidth product $T B=M N$.

Now, constructing an $(L / M) \times N$ matrix $\boldsymbol{\Phi}(m)$ as

$$
\begin{aligned}
{[\Phi(m)]_{\tilde{l}, n}=} & e^{j 2 \pi\left(n-\frac{N+1}{2}\right)\left(\tilde{l}+(m-1) \frac{L}{M}-1\right)}, \\
& \text { for } \tilde{l}=1,2, \ldots,(L / M) \text { and } n=1,2, \ldots N,
\end{aligned}
$$

we can rewrite (13) in the form of (12) as

$$
s_{\mathrm{MCPC}}=\boldsymbol{\Phi}_{\mathrm{MCPC}} a \text {, }
$$

where $\boldsymbol{\Phi}_{\mathrm{MCPC}}=\operatorname{blkdiag}(\boldsymbol{\Phi}(1), \boldsymbol{\Phi}(2), \ldots, \boldsymbol{\Phi}(M))$ is an $L \times$ $M N$ block-diagonal matrix that constitutes the family of MCPC waveforms; and $\boldsymbol{a}=\left[a_{1,1}, \ldots, a_{1, N}, a_{2,1}, \ldots, a_{M, N}\right]^{T}$ is an $M N \times 1$ phase-code vector.

Then, using (9), the spectrum of an MCPC signal is easily expressed as

$$
S_{\mathrm{MCPC}}\left(f_{k}\right)=\boldsymbol{w}_{k}^{H} \boldsymbol{\Phi}_{\mathrm{MCPC}} \boldsymbol{a},
$$

which ultimately reduces to

$$
\begin{aligned}
S_{\mathrm{MCPC}}\left(f_{k}\right)= & \sum_{m=1}^{M} \sum_{n=1}^{N} a_{m, n} t_{\mathrm{b}} \operatorname{sinc}\left(\left(f_{k}-\tilde{n} f_{\mathrm{b}}\right) t_{\mathrm{b}}\right) \\
& \times e^{-j \pi\left(f_{k}-\tilde{n} f_{\mathrm{b}}\right) t_{\mathrm{b}}} e^{-j 2 \pi(m-1)\left(f_{k}-\tilde{n} f_{\mathrm{b}}\right) t_{\mathrm{b}}},
\end{aligned}
$$

where $\tilde{n} \equiv n-(N+1) / 2$ and $f_{\mathrm{b}}=1 / t_{\mathrm{b}}$. Therefore, when the MCPC signaling technique is used, the performance of an optimal target detection algorithm is given by

$$
\begin{aligned}
& d_{\mathrm{MCPC}}(\boldsymbol{a})^{2}= \\
& \sum_{k=-K / 2}^{K / 2-1} \frac{\boldsymbol{a}^{H}\left[\sigma_{\mathrm{x}}^{2}\left|G_{\mathrm{T}}\left(f_{k}\right)\right|^{2} \boldsymbol{\Phi}_{\mathrm{MCPC}}^{H} \boldsymbol{w}_{k} \boldsymbol{w}_{k}^{H} \boldsymbol{\Phi}_{\mathrm{MCPC}}\right] \boldsymbol{a}}{\left.\mathcal{P}_{\mathrm{c}}\left(f_{k}\right) \boldsymbol{\Phi}_{\mathrm{MCPC}}^{H} \boldsymbol{w}_{k} \boldsymbol{w}_{k}^{H} \boldsymbol{\Phi}_{\mathrm{MCPC}}\right] \boldsymbol{a}+\mathcal{P}_{\mathrm{e}}\left(f_{k}\right)} \Delta f,
\end{aligned}
$$

and we obtain the optimal phase-codes of an MCPC waveform $s_{\mathrm{MCPC}}$ by maximizing $d^{2}$ with respect to $\boldsymbol{a}$ as

$$
\begin{aligned}
\boldsymbol{a}_{\mathrm{opt}}= & \arg \max _{\boldsymbol{a} \in \mathbb{C}^{M N}} d_{\mathrm{MCPC}}(\boldsymbol{a})^{2} \\
& \text { subject to } \boldsymbol{a}^{H} \mathbf{\Phi}_{\mathrm{MCPC}}^{H} \boldsymbol{\Phi}_{\mathrm{MCPC}} \boldsymbol{a}=\varepsilon / t_{\mathrm{s}} .
\end{aligned}
$$

To obtain a comparative performance analysis of the designed MCPC waveforms with an existing waveform design technique, we study [12] which synthesizes a transmit signal based on the discrete prolate spheroidal sequences (DPSS) as

$$
s_{\mathrm{DPSS}}(l)=\sum_{\substack{r=0 \\ \text { for } l=0,1, \ldots, L-1,}}^{2 L B_{\mathrm{n}}-1} c_{r} v_{r}\left(l ; L, B_{\mathrm{n}}\right),
$$

where $c=\left[c_{0}, c_{1}, \ldots, c_{\left(2 L B_{\mathrm{n}}-1\right)}\right]^{T}$ represents a weight vector and $\left\{v_{r}\left(l ; L, B_{\mathrm{n}}\right), l=0,1, \ldots, L-1\right\}$ denotes the $r$ th order DPSS of length $L$ and normalized bandwidth $B_{\mathrm{n}}$. Therefore, when $B_{\mathrm{n}}=0.5$ the dimension of the weight vector $c$ becomes $L$ which is at least twice the dimension of the MCPC code vector $\boldsymbol{a}$.

Now, forming an $L \times\left(2 M B_{\mathrm{n}}-1\right)$ matrix $\boldsymbol{\Phi}_{\mathrm{DPSS}}$ whose $r$ th column contains the $r$ th order DPSS $\left[v_{r}\left(0 ; L, B_{\mathrm{n}}\right), \ldots, v_{r}(L-\right.$ $\left.\left.1 ; L, B_{\mathrm{n}}\right)\right]^{T}$, we can rewrite (19) as

$$
s_{\mathrm{DPSS}}=\Phi_{\mathrm{DPSS}} c .
$$

Next, the optimal waveform $s_{\text {DPSS }}$ is obtained by solving for the optimal value of $c$ that maximizes the detection performance as

$$
\begin{aligned}
\boldsymbol{c}_{\mathrm{opt}}= & \arg \max _{\boldsymbol{c}} d_{\mathrm{DPSS}}(\boldsymbol{c})^{2} \\
& \text { subject to } \boldsymbol{c}^{H} \boldsymbol{\Phi}_{\mathrm{DPSS}}^{H} \boldsymbol{\Phi}_{\mathrm{DPSS}} \boldsymbol{c}=\varepsilon / t_{\mathrm{S}},
\end{aligned}
$$

where

$$
\begin{aligned}
& d_{\mathrm{DPSS}}(\boldsymbol{c})^{2}= \\
& \sum_{k=-K / 2}^{K / 2-1} \frac{\boldsymbol{c}^{H}\left[\sigma_{\mathrm{x}}^{2}\left|G_{\mathrm{T}}\left(f_{k}\right)\right|^{2} \boldsymbol{\Phi}_{\mathrm{DPSS}}^{H} \boldsymbol{w}_{k} \boldsymbol{w}_{k}^{H} \boldsymbol{\Phi}_{\mathrm{DPSS}}\right] \boldsymbol{c}}{\left.\mathcal{P}_{\mathrm{c}}\left(f_{k}\right) \boldsymbol{\Phi}_{\mathrm{DPSS}}^{H} \boldsymbol{w}_{k} \boldsymbol{w}_{k}^{H} \boldsymbol{\Phi}_{\mathrm{DPSS}}\right] \boldsymbol{c}+\mathcal{P}_{\mathrm{e}}\left(f_{k}\right)} \Delta f .
\end{aligned}
$$

\section{Numerical Results}

In this section, we present the results of several numerical examples to demonstrate the performance of the designed MCPC signals. The target, clutter, and noise PSDs were simulated following the examples of [11]. Here, we briefly summarize the simulation setup.

- Interference parameters: In Scenario I, we took a flat clutter-PSD $\mathcal{P}_{\mathrm{c}}^{(1)}(f)=1$, for which the clutter return 

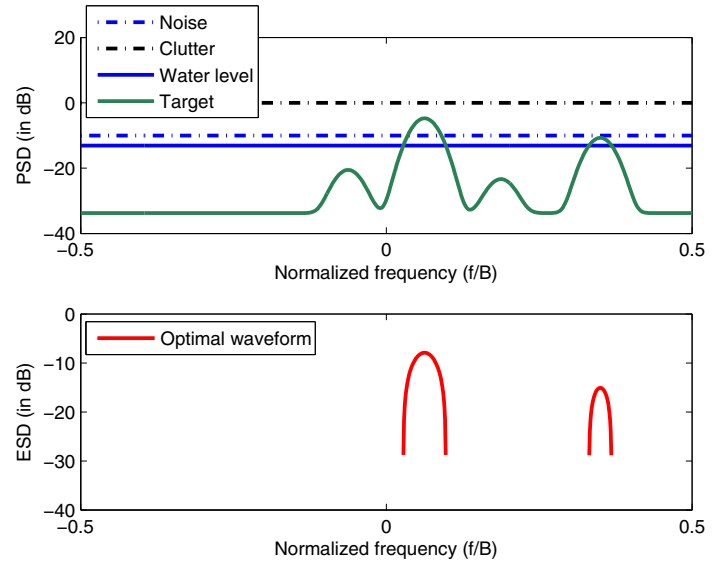

(a)
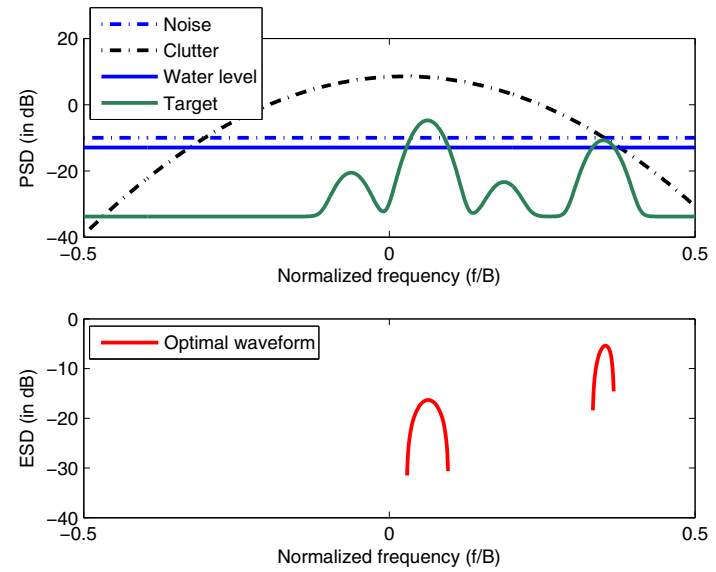

(b)

Fig. 2. Variations of the target, clutter, noise powers, and the optimal-signal energies in (a) Scenario I and (b) Scenario II.

would be just a scaled version of the transmitted-signal ESD. In Scenario II, we modeled the clutter PSD to have a Gaussian shape: $\mathcal{P}_{\mathrm{c}}^{(2)}(f)=e^{-\left(f-f_{\mathrm{c}}\right)^{2} /\left(2 \sigma_{\mathrm{c}}^{2}\right)}$, where $f_{\mathrm{c}}=0.2 \mathrm{kHz}$ and $\sigma_{\mathrm{c}}^{2}=8 \times 10^{5}$. The noise PSDs were assumed to be flat in both the simulated scenarios.

- Target parameters: In both the scenarios, we modeled the target signature as a Gaussian mixer:

$$
\left|G_{\mathrm{T}}(f)\right|^{2}=g_{0}+\sum_{i=1}^{4} g_{i} e^{\left(-\frac{\left(f-f_{i}\right)^{2}}{2 \sigma_{\mathrm{T}}^{2}}\right)},
$$

where $g_{0}=0.001, g_{1}=0.8, g_{2}=0.01, g_{3}=0.2$, $g_{4}=0.02 ;$ the frequencies were $f_{1}=0.5 \mathrm{kHz}, f_{2}=$ $1.5 \mathrm{kHz}, f_{3}=2.8 \mathrm{kHz}, f_{4}=-0.5 \mathrm{kHz}$; and variance was $\sigma_{\mathrm{T}}^{2}=2 \times 10^{4}$.

- Simulation parameters: We considered the total energy of $\mathcal{P}_{\mathrm{c}}^{(1)}(f)$ over a bandwidth of $8 \mathrm{kHz}$ as the baseline energy measure. Then, we scaled $\mathcal{P}_{c}^{(2)}(f)$ to ensure that the total clutter-energy remain the same, i.e., $\sum_{n} \mathcal{P}_{\mathrm{c}}^{(1)}\left(f_{n}\right)=$ $\sum_{n} \mathcal{P}_{\mathrm{c}}^{(2)}\left(f_{n}\right)$. Thereafter, we scaled the target-signatures and noise-PSDs of the respective scenarios to satisfy the
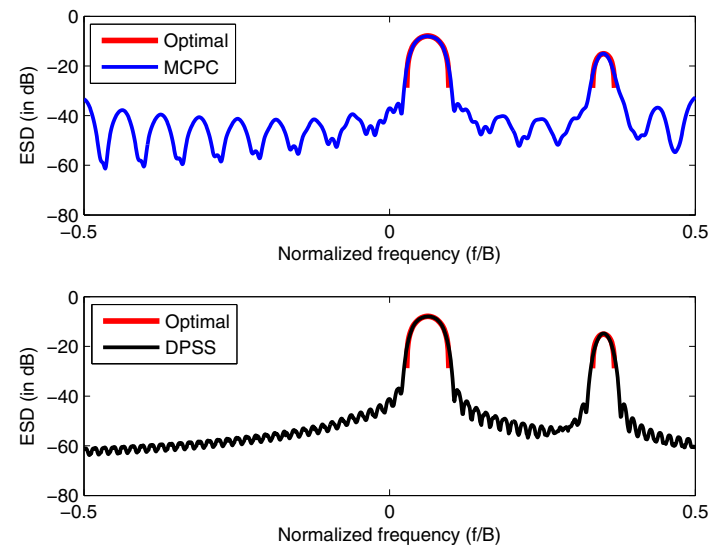

(a)
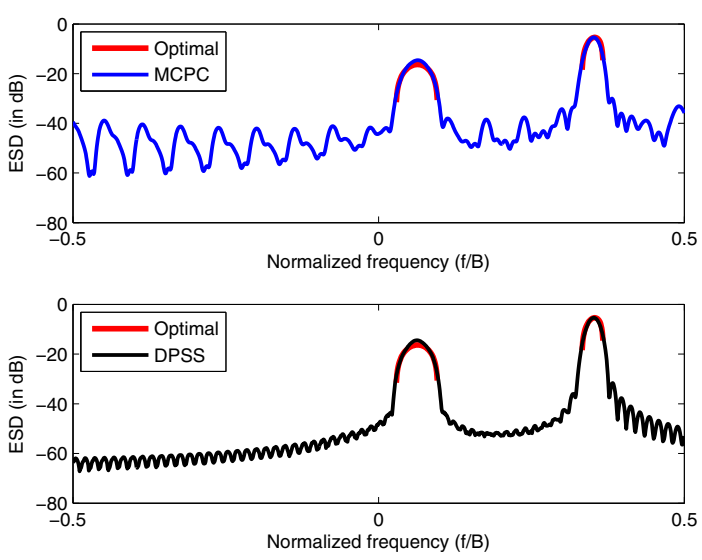

(b)

Fig. 3. Energy spectral densities of the designed MCPC and DPSS waveforms in (a) Scenario I and (b) Scenario II.

clutter-to-noise ratio of $10 \mathrm{~dB}$ and signal-to-noise ratio of $-10 \mathrm{~dB}$. The transmit signals were generated with a pulse duration $T=8 \mathrm{~ms}$ and the total energy $\varepsilon=30$.

The MCPC waveforms were simulated with $M=4$ and $N=16$, implying the inter-carrier frequency spacing $f_{\mathrm{b}}=500 \mathrm{~Hz}$, and the DPSS signals had $B_{\mathrm{n}}=0.5$.

Fig. 2 depicts the PSDs of target, clutter, and noise processes, and the resultant optimal-ESDs obtained from (8) in both the scenarios. In Scenario I, the water level $\lambda \mathcal{P}_{\mathrm{e}}(f)$ was at $-13.09 \mathrm{~dB}$, and therefore the optimal-ESD was nonzero over two discrete frequency-bands $(0.028,0.097)$ and $(0.332,0.368)$. Relative to the clutter-plus-noise power, the target power at the first frequency-band was stronger than that at the second frequency-band. Therefore, from Fig. 2(a) we notice that the optimal-ESD put more signal energy in the first frequency-band than the second. On the other hand, in Scenario II, we got the nonzero optimal-ESD over two similar frequency-bands $(0.029,0.096)$ and $(0.333,0.367)$ corresponding to the water level $\lambda \mathcal{P}_{\mathrm{e}}(f)=-12.93 \mathrm{~dB}$; but the signal energy at the first frequency-band was weaker 

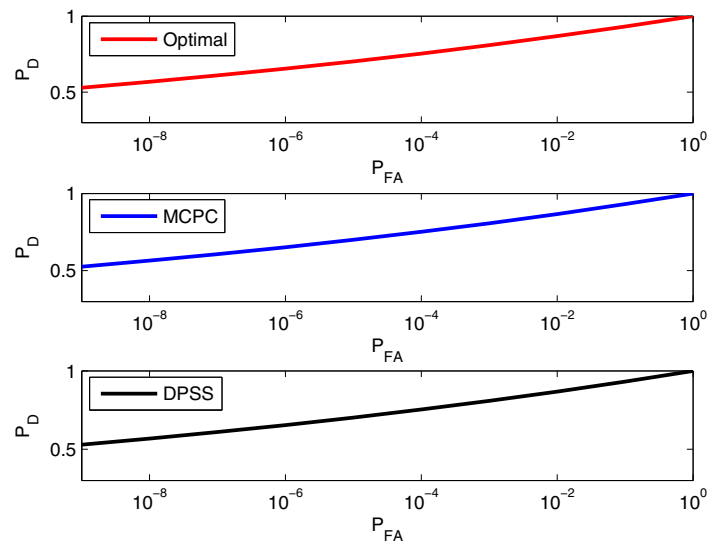

(a)
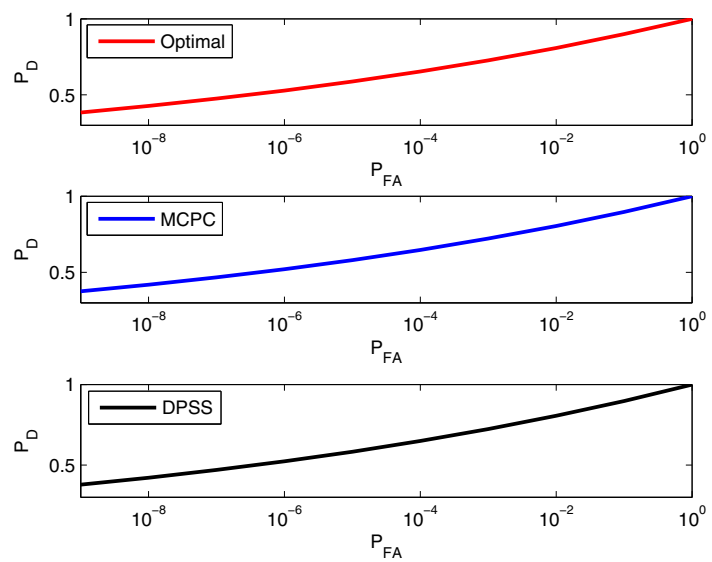

(b)

Fig. 4. Receiver operating characteristics of the optimal, DPSS, and MCPC waveforms in (a) Scenario I and (b) Scenario II.

than that at the second due to the change in target-to-clutterplus-noise ratios at these two bands. In addition, the overall detection performances in Scenarios I and II were found to be $d^{2}=31.60$ and 20.66 respectively.

Fig. 3(a) shows the ESD of the designed MCPC waveform in Scenario I. In the same figure, we also plot the optimal-ESD and the ESD of DPSS waveform. We noticed that the ESD of the MCPC signal well-matched with the optimal-ESD at the frequencies where the target powers were stronger than the water level; however, MCPC-ESD had relatively higher signal energies than the DPSS-ESD along those frequencies in which the target powers were weaker than the water level. In Scenario II, we found that the ESD of the MCPC signal was very similar to the optimal- and DPSS-ESDs particularly when the target response was strong, as shown in Fig. 3(b).

Overall, in terms of the $d^{2}$ values as shown in Table I, the MCPC signals produced the near-optimal performances as well as the DPSS waveforms. We plot the resultant detection performance in terms of the receiver operating characteristics (ROC) in Fig. 4. In both the Scenarios I and II, the ROC
TABLE I

DETECTION PERFORMANCES OF THE DESIGNED MCPC AND DPSS WAVEFORMS

\begin{tabular}{lcc}
\hline \hline Waveforms & $\begin{array}{c}\text { Values of } d^{2} \\
\text { in Scenario I }\end{array}$ & $\begin{array}{c}\text { Values of } d^{2} \\
\text { in Scenario II }\end{array}$ \\
\hline Optimal & 31.60 & 20.66 \\
MCPC & 31.36 & 20.39 \\
DPSS & 31.54 & 20.55 \\
\hline \hline
\end{tabular}

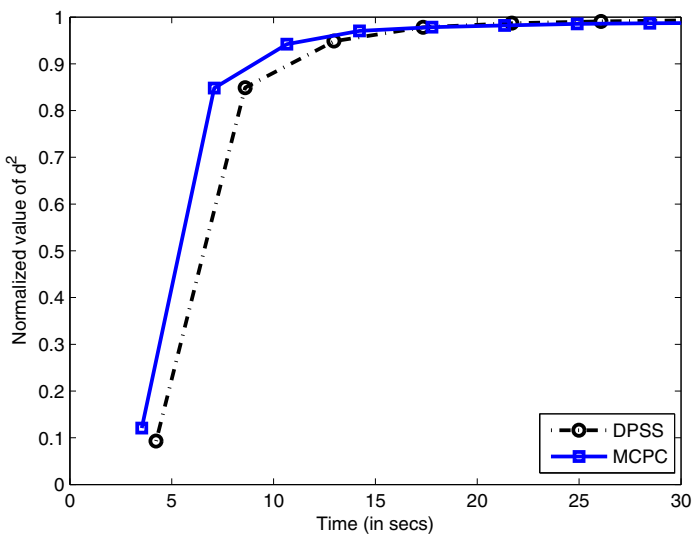

(a)

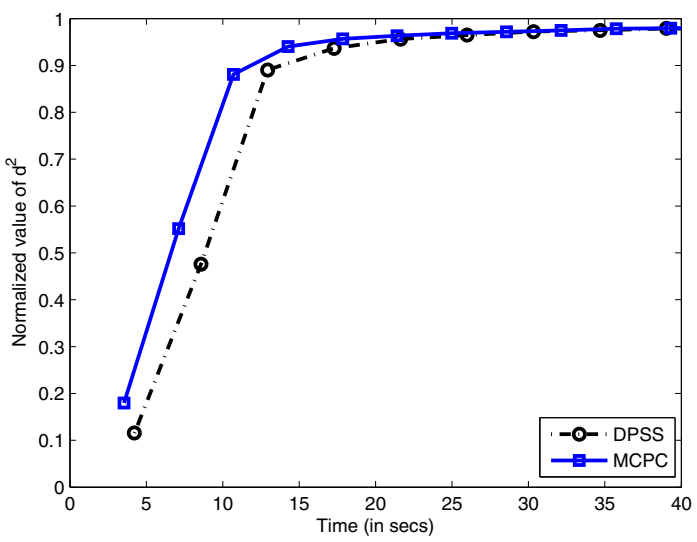

(b)

Fig. 5. Comparative convergence rates of the DPSS and MCPC waveform designs in (a) Scenario I and (b) Scenario II.

plots of all the three designed waveforms (optimal, MCPC, and DPSS) were so exact that we could not show them on a single plot and had to use subplots. This confirms that the detection performance of the designed MCPC waveform is very similar to the optimal performance.

Importantly, we designed the MCPC signals by solving an $M N$ dimensional optimization problem which was about half in size than the DPSS based optimization problem involving $L$ dimensional design vector. Therefore, from Fig. 5, we observe that the computational-time required for the design of MCPC signal was approximately $10-15 \%$ less than that required for the DPSS signal. 


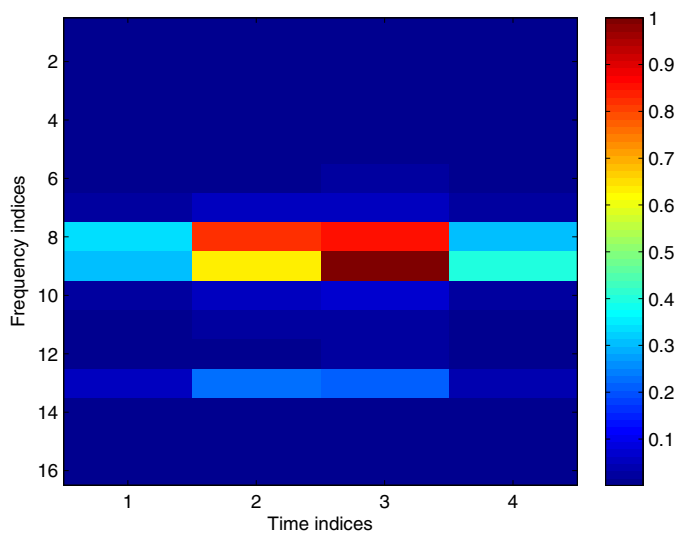

(a)

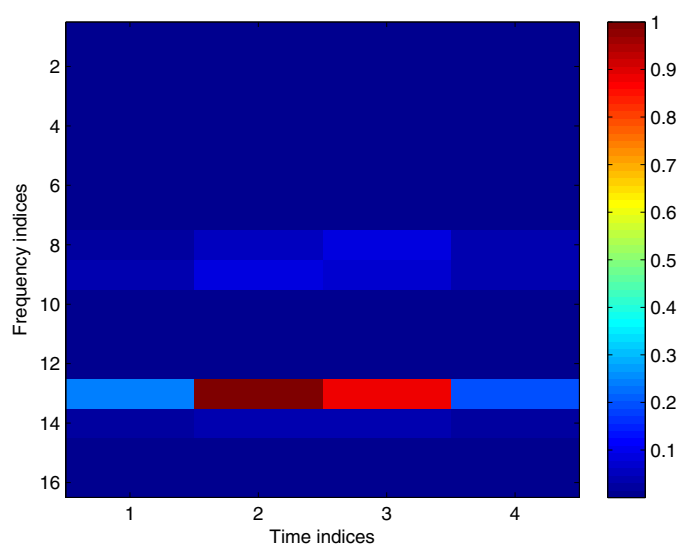

(b)

Fig. 6. Structures of the designed MCPC signals in (a) Scenario I and (b) Scenario II.

The code-structures of the designed MCPC signals are demonstrated in Fig. 6 for both the scenarios. From these plots, we can easily understand how the energies of the designed codes are distributed among different time and frequency bins. For example, in Scenario I the first nonzero frequencyband $(0.028,0.097)$ of the optimal-ESD corresponded to the 8th and 9th frequency-bins of the MCPC signals, with the strongest signal energy occurring at the 9th frequency-bin. Similarly, in Scenario II, the strongest signal energy was at the 13th frequency-bin that matched with the second nonzero frequency-band $(0.333,0.367)$ of the optimal ESD.

\section{COnClusions}

In this paper, we developed a direct time-domain approach to design a parametric multicarrier phase-coded (MCPC) waveform that attained the optimal performance in detecting an extended target. The time-domain waveform synthesis technique gave us a straightforward way to analyze the perfor- mance of the designed signal bypassing the requirement of the optimal energy spectral density computation. First, we expressed the detection characteristics in terms of the phase codes of the MCPC waveforms, and then optimally designed an MCPC signal by maximizing the detection performance with respect to the phase codes. We presented a comparative performance analysis of our designed MCPC signals with another existing waveform design technique based on the discrete prolate spheroidal sequences (DPSS). Our numerical results demonstrated that the MCPC waveforms had a near-optimum detection performance and required a lesser computationaltime than the DPSS signals. In our future work, we intend to analyze the performances of the designed signals with respect to their autocorrelation and peak-to-average power ratio properties.

\section{REFERENCES}

[1] M. I. Skolnik, Introduction to Radar Systems, 3rd ed. New York, NY: McGraw-Hill, Dec. 2002

[2] M. C. Wicks, "A brief history of waveform diversity," in Proc. IEEE Radar Conf., Pasadena, CA, May 4-8, 2009, pp. 1-6.

[3] A. Nehorai, F. Gini, M. S. Greco, A. P. Suppappola, and M. Rangaswamy, "Introduction to the issue on adaptive waveform design for agile sensing and communication," IEEE J. Sel. Topics Signal Process., vol. 1 , no. 1, pp. 2-5, Jun. 2007.

[4] R. Calderbank, S. Howard, and B. Moran, "Waveform diversity in radar signal processing," IEEE Signal Process. Mag., vol. 26, no. 1, pp. 32-41, Jan. 2009.

[5] M. C. Wicks, E. Mokole, S. Blunt, R. Schneible, and V. Amuso, Eds., Principles of Waveform Diversity and Design. SciTech Pub., Aug. 2010.

[6] F. Gini, A. De Maio, and L. K. Patton, Waveform Design and Diversity for Advanced Radar Systems. Inst of Engineering \& Technology, Jun. 2011.

[7] M. Bell, "Information theory and radar waveform design," IEEE Trans. Inf. Theory, vol. 39, no. 5, pp. 1578-1597, Sep. 1993.

[8] S. Kay, "Optimal signal design for detection of Gaussian point targets in stationary Gaussian clutter/reverberation," IEEE J. Sel. Topics Signal Process., vol. 1, no. 1, pp. 31-41, Jun. 2007.

[9] R. Romero and N. Goodman, "Waveform design in signal-dependent interference and application to target recognition with multiple transmissions," IET Radar, Sonar Navigation, vol. 3, no. 4, pp. $328-340$, Aug. 2009.

[10] R. Romero, J. Bae, and N. Goodman, "Theory and application of SNR and mutual information matched illumination waveforms," IEEE Trans. Aerosp. and Electron. Syst., vol. 47, no. 2, pp. 912 -927, Apr. 2011.

[11] X. Deng, C. Qiu, Z. Cao, M. Morelande, and B. Moran, "Waveform design for enhanced detection of extended target in signal-dependent interference," IET Radar, Sonar Navigation, vol. 6, no. 1, pp. $30-38$, Jan. 2012.

[12] F. Yin, C. Debes, and A. Zoubir, "Parametric waveform design using discrete prolate spheroidal sequences for enhanced detection of extended targets," IEEE Trans. Signal Process., vol. 60, no. 9, pp. 4525 -4536, Sep. 2012

[13] X. Gong, H. Meng, Y. Wei, and X. Wang, "Phase-modulated waveform design for extended target detection in the presence of clutter," Sensors, vol. 11, no. 7, pp. 7162-7177, Jul. 2011.

[14] N. Levanon, "Multifrequency complementary phase-coded radar signal," IEE Proc. - Radar, Sonar and Nav., vol. 147, no. 6, pp. 276 -284, Dec. 2000.

[15] _ "Multifrequency radar signals," in Proc. Int. Radar Conf., Alexandria, VA, May 7-12, 2000, pp. $683-688$.

[16] N. Levanon and E. Mozeson, Radar Signals. Hoboken, NJ: John Wiley \& Sons, Jul. 2004. 